\title{
The Effect of HIV and Antiretroviral Therapy on Chromosomal Radiosensitivity
}

\author{
Olivia Herd $^{1,2}$, Flavia Francies ${ }^{1,2}$, Jacobus Slabbert ${ }^{1}$ and Ans Baeyens $\mathbf{1}^{1,2^{*}}$ \\ ${ }^{1}$ Department of Radiation Biophysics, iThemba Labs, Somerset West, South Africa \\ ${ }^{2}$ Radiobiology, Department of Radiation Sciences, University of Witwatersrand, Johannesburg, South Africa
}

*Corresponding author: Ans Baeyens, Department of Radiation Biophysics, iThemba LABS and Department of Radiation Sciences, University of Witwatersrand, Area 348, Orange Block, 4th floor, Charlotte Maxeke Academic Hospital, Jubilee Road, Parktown 2193, Johannesburg, South Africa, Tel: +27 (11) 4812160; Fax: +27 (11) 4849202; Email: Ans.Baeyens@wits.ac.za; ansbaeyens@hotmail.com

Received date: October 14, 2014; Accepted date: November 27, 2014, Published date: December 02, 2014

Copyright: (C) 2014 Herd O, et al. This is an open-access article distributed under the terms of the Creative Commons Attribution License, which permits unrestricted use, distribution, and reproduction in any medium, provided the original author and source are credited.

\begin{abstract}
Introduction: Antiretroviral Treatment (ART) has led to an improvement in survival of HIV infected individuals. Some of them will develop cancer during the course of their infection and will require radiation therapy. HIV positive cancer patients have presented with adverse side effects of radiotherapy and elevated chromosomal radiosensitivity. This study investigated if ART has an influence on chromosomal radiosensitivity of HIV positive individuals.

Methods and Materials: Blood samples from 60 HIV positive individuals were in vitro exposed to doses of X-rays of 0,2 and $4 \mathrm{~Gy}$ and chromosomal radiosensitivity was assessed with the micronucleus assay. The micronucleus assay was also performed on lymphocytes of a group of non HIV-infected health care workers taking prophylactic post-exposure ART to measure the effect of these ART drugs on chromosomal radiosensitivity without HIV as a confounding factor.
\end{abstract}

Results: All HIV patients (those on ART and without ART) had significantly higher radiation induced Micronuclei (MN) than healthy controls. The MN yields increased in the HIV patients taking ART compared to HIV patients not on treatment. The evaluation of chromosomal radiosensitivity of health care workers on ART revealed no effects of ART.

Conclusions: HIV positive individuals show an increased chromosomal radiosensitivity. Antiretroviral treatment given to HIV positive individuals can lead to enhanced chromosomal radiosensitivity and therefore impose higher risks for radiotherapy side effects in these patients.

Keywords: Antiretroviral treatment; HIV; Chromosomal radiosensitivity

\section{Introduction}

In 2013, there were 5.6 million people living with Human Immunodeficiency Virus (HIV) in South Africa [1]. Antiretroviral Therapy (ART) has led to the improvement in health and survival of patients with HIV [2]. South Africa has one of the largest ART rollouts in the world and in 2013 over 2 million South Africans were on ART [2]. The 2006 World Health Organisation's guidelines for treatment of HIV infection in adults recommended a first-line regimen of two Nucleoside Reverse Transcriptase Inhibitors (NRTIs) combined with one Non-Nucleoside Reverse Transcriptase Inhibitor (NNRTI) [3]. Due to their cost effectiveness, Stavudine (d4T) and Zidovudine (AZT) were the most commonly prescribed NRTIs in low and middle income countries [4], however due its toxicity, the WHO updated guidelines recommended using Tenofovir (TDF) instead of $\mathrm{d} 4 \mathrm{~T}$ in first-line therapy [5]. In 2010 the South African Department of Health changed it guidelines to recommend TDF+lamivudine (3TC)/ emtricitabine (FTC)+efavirenz (EFV) as first-line therapy for all new HIV patients and for those on $\mathrm{d} 4 \mathrm{~T}+3 \mathrm{TC} / \mathrm{FTC}+\mathrm{EFV}$ without toxicity to remain on that regimen [6]. According to the WHO 2013 guidelines [7] all patients should be switched to the TDF-based regimen and the transition of patients to a fixed-dose combination one-pill ART regimen containing TDF+FTC+EFV began in South Africa in 2013 [8].

ART is not only used in treatment of HIV but also in prevention. ART is administered prophylactically to non-infected persons after occupational exposure to HIV. A study in Health Care Workers (HCWs) has shown post-exposure prophylaxis to be protective after exposure to the HIV virus [9]. With the high incidence of HIV in South Africa, HCWs face constant risk of being exposed to HIV after being cut or pricked by sharps during routine patient care. A South African study showed that $69 \%$ of medical interns reported at least 1 percutaneous injury during their year-long internship [10]. The WHO guidelines recommend a combination of antiretroviral drugs for postexposure prophylaxis based on two nucleoside reverse transcriptase inhibitors [11].

HIV infection is associated with a high risk of developing specific AIDS-related cancers such as non-Hodgkin lymphoma, Kaposi sarcoma and cervical cancer [12]. The prolonged survival of HIV infected individuals, largely owing to improved ART access, has also led to an increasing incidence of non-AIDS related cancers amongst these individuals $[13,14]$. Radiotherapy is a mainstay treatment for cancers in South Africa where cancer patients, many of which will be HIV positive, often present with advanced disease due to limited access to diagnostic centers in rural areas, lack of awareness and lower standards of healthcare facilities. As South Africa is also a country with 
a prolific radiation industry and many radiation workers, the high number of HIV infected people and taking ART in the general South African population is also likely to be reflected in this workforce. The effect of HIV and ART on chromosomal radiosensitivity can have important implications for radiation protection in these workers.

A pilot study by Baeyens et al. (2010) has previously shown HIV positive patients to be more chromosomally radiosensitive than HIV negative controls [15]. Chromosomal radiosensitivity is increased vulnerability of cells to the DNA-damaging effects of ionizing radiation. It can be a result of impaired DNA damage repair. Associations between clinical response to radiotherapy and chromosomal radiosensitivity $[16,17]$ and elevated radiotoxicity such as mucositis and neutropenia has been reported in some HIV patients receiving radiotherapy for several types of cancers (reviewed in [18]). As radiotherapy decreases CD4 cell counts [19], HIV positive people in clinical settings are advised to start ART before undergoing radiotherapy to minimise the risk of developing AIDS, particularly when the patient has low $\mathrm{CD} 4$ counts.

Since NRTIs and NNRTIs interact with host DNA, it is possible that they could have an impact on DNA damage repair and affect chromosomal radiosensitivity. AZT, 3TC and TDF have been shown to inhibit cell growth [20], to interfere with cell cycle arrest, induce cell death by apoptosis and inhibit telomerase activity [21-24]. Conflicting studies have shown AZT to act as a radioprotector or a radiosensitizer $[25,26]$.

Chromosomal radiosensitivity can be tested through a variety of cytogenetic assays. A reliable and well-established method is the cytokinesis-block micronucleus assay ( $\mathrm{MN}$ assay) that is commonly used in biological dosimetry and chromosomal radiosensitivity studies. Micronuclei (MN) are small nuclei that form in the cytoplasm when chromosomes or chromosome fragments are not incorporated into the daughter nuclei subsequent to cell division [27]. MN reflect the amount of DNA damage and DNA damage repair after exposure to radiation. The MN assay can be performed on lymphocytes, which are easily obtainable via venipuncture. The micronucleus assay is also considered a sensitive method to evaluate the cytotoxic potential of an active agent. The nucleoside reverse transcriptase inhibitors AZT, 3TC and $\mathrm{d} 4 \mathrm{~T}$ have been shown to produce an increase in $\mathrm{MN}$ in human lymphocyte cultures [28-30].

In this study the micronucleus assay on the lymphocytes of HIV patients not taking ART and of HIV patients on one of the 2 most commonly administered ART regimens in South Africa $(\mathrm{d} 4 \mathrm{~T}+3 \mathrm{TC}$ $+\mathrm{EFV}$ and TDF+3TC+EFV) was performed to assess the effect of HIV and these drugs on chromosomal radiosensitivity. The micronucleus assay on the lymphocytes of a group of non HIV-infected health care workers taking prophylactic post-exposure ART was also performed to measure the effect of these ART drugs on chromosomal radiosensitivity without HIV as a confounding factor.

\section{Materials and Methods}

\section{Study population}

Heparinised blood samples from 60 HIV positive individuals were obtained from the Themba Lethu HIV unit at the Helen Joseph Hospital in Johannesburg, South Africa. Twenty participants were HIV positive but not taking any ART medication. Twenty participants were on the $\mathrm{d} 4 \mathrm{~T}+3 \mathrm{TC}+\mathrm{EFV}(30 \mathrm{mg} / 150 \mathrm{mg} / 600 \mathrm{mg})$ regimen and twenty participants were taking the TDF+3TC+EFV $(300 \mathrm{mg} / 150 \mathrm{mg} /$ $600 \mathrm{mg}$ ) regimen. HIV patients were on ART for at least 6 months. Blood samples of $20 \mathrm{HIV}$ negative healthy individuals, age-matched with HIV positive individuals, were included as controls. These were staff members and students from Charlotte Maxeke Johannesburg Academic hospital (CMJAH) where the study was undertaken. Population characteristics and treatment data are shown in Table 1. The CD4 counts, measured on the same day as the MN assay, were extracted from clinical records.

\begin{tabular}{|c|c|c|c|c|c|}
\hline & & & HIV ART & HIV ART & \\
\hline & Healthy individuals & HIV without ART & D4T/3TC/EFV & TDF/3TC/EFV & Health care workers \\
\hline \multicolumn{6}{|l|}{ Gender } \\
\hline female/male & $14 / 6$ & $15 / 5$ & $13 / 7$ & $18 / 2$ & $8 / 2$ \\
\hline \multicolumn{6}{|l|}{ Age } \\
\hline mean \pm SD & $30 \pm 7$ & $36 \pm 10$ & $37 \pm 7$ & $41 \pm 6$ & $31 \pm 11$ \\
\hline range (min-max) & $23-52$ & $18-55$ & $29-54$ & $34-53$ & $22-56$ \\
\hline \multicolumn{6}{|l|}{ CD4 cell counts } \\
\hline mean \pm SD & & $480 \pm 164$ ** & $623 \pm 240$ & $649 \pm 204$ & \\
\hline range & $(500-1200)^{*}$ & $268-858$ & $202-1081$ & $302-933$ & \\
\hline \multicolumn{6}{|c|}{ Duration of ART (months) } \\
\hline mean $\pm S D$ & & & $51 \pm 26$ & $51 \pm 28$ & 1 \\
\hline range & & & $13-100$ & $6-99$ & \\
\hline
\end{tabular}


Table 1: Patient Characteristics.

For the second part of the study, heparinised blood samples of 10 health care workers on post-exposure ART prophylaxis were collected from CMJAH. All the HCWs were taking a combination of 2 NRTIs (300mg AZT + 150mg 3TC or 200mg FTC + 300mg TDF) for 28 days. Blood samples of these HCWs were taken at 3 time points: The first was the day of the needle-stick or sharps injury, just before starting prophylaxis treatment (T1); the second was 3-4 weeks after T1, after completion of the ART course of medication (T2); the third was between 3 and 4 months after T1 (T3). All blood donors signed informed consent and the study was approved by the Human Research Ethics Committee, University of Witwatersrand, Johannesburg, South Africa (M110361 and M120270).

\section{Micronucleus assay}

The micronucleus assay was performed as described by Baeyens et al. [15]. In vitro chromosomal radiosensitivity was measured by scoring MN in lymphocytes irradiated with doses of 2 Gray (Gy) and 4 Gy. A 2 Gy dose is frequently used in radiotherapy fractionation schedules. A 0 Gy dose was used as a sham-irradiated control (spontaneous MN) and also allowed us to examine the cytotoxic effect of ART on lymphocytes without irradiation. Lymphocyte cultures were set up by adding $0.5 \mathrm{ml}$ blood to $4.5 \mathrm{ml}$ of prewarmed RPMI (Roswell Park Memorial Institute) 1640 (BioWhittaker, Walkersville, USA) that was supplemented with $13 \%$ foetal bovine serum (GibcoInvitrogen, New York, USA) and $0.01 \%$ antibiotics (50 U/ml penicillin and $50 \mathrm{mg} / \mathrm{ml}$ streptomycin; Gibco-Invitrogen). The irradiations were done in the Radiation Oncology Unit at CMJAH. Culture flasks were placed in a Phantom-water tank at room temperature and irradiated with X-rays using a $6 \mathrm{MV}$ photon beam from a medical linear accelerator (Siemens Healthcare, Erlangen, Germany). The distance from the culture flasks to the radiation source was $100 \mathrm{~cm}$ at an angle of 90 degrees. The field size at the depth of the sample was $10 \mathrm{X} 10 \mathrm{~cm}$ and the dose rate was approximately $1.33 \mathrm{~Gy} / \mathrm{min}$. For each dose point, 2 co-cultures were set up. Subsequent to irradiation, lymphocytes were stimulated by adding $100 \mu \mathrm{l}$ of phytohaemagglutinin (stock solution $1 \mathrm{mg} / \mathrm{ml}$, Sigma-Aldrich, St Louis, MO, USA). To block cytokinesis, $20 \mu \mathrm{l}$ cytochalasin B (stock solution of $1.5 \mathrm{mg} / \mathrm{ml}$, Sigma-Aldrich) was added to the cultures at $23 \mathrm{hrs}$. Cells were harvested $70 \mathrm{hrs}$ after stimulation using a cold $\left(4^{\circ} \mathrm{C}\right)$ hypotonic shock with $7 \mathrm{ml} 0.075 \mathrm{M} \mathrm{KCl}$ (Merck, Darmstadt, Germany). This was followed by fixation in methanol: acetic acid: Ringer $(0.9 \%$ $\mathrm{NaCl})$ solution (4:1:5) (Merck) at $4^{\circ} \mathrm{C}$ overnight. Thereafter cells were fixed another three times with methanol: acetic acid (4:1) (Merck). Cell suspensions were dropped on slides and stained for 1 minute with acridine orange stain $(10 \mu \mathrm{g} / \mathrm{ml})$ (Sigma-Aldrich, St Louis, MO, USA) and immersed for $1 \mathrm{~min}$ in acridine orange buffer (Sigma-Aldrich, St Louis, MO, USA). Duplicate slides were made of each sample, coded and scored blindly by 2 experienced scorers using a Zeiss Axioskop fluorescent microscope (400X) (Carl Zeiss, Gottingen, Germany). At least 500 binucleated cells $(\mathrm{BN})$ were scored per slide, according to the criteria of Fenech [31]. The results of both scorers were combined and normalised to $1000 \mathrm{BN}$. The number of mononucleated $\left(\mathrm{N}_{1}\right)$, binucleated $\left(\mathrm{N}_{2}\right)$, trinucleated $\left(\mathrm{N}_{3}\right)$ and polynucleated $\left(\mathrm{N}_{4}\right)$ cells per slide was scored and the nuclear division index (NDI) was calculated according to the formula: $\mathrm{NDI}=\left(\mathrm{N}_{1}+2 \mathrm{~N}_{2}+3 \mathrm{~N}_{3}+4 \mathrm{~N}_{4}\right) / \mathrm{N}$ tot where Ntot is the total number of cells per slide counted (Ntot $=500$ cells).

\section{Statistical analysis}

Statistical analysis was performed with Graph Pad Prism 6. The non-parametric Mann-whitney test was used to compare means of MN counts and NDI values between the different population groups. For HCWs, differences between MN counts at different time points were tested for significance with the non-parametric Wilcoxon test. Significance was set at $\mathrm{p}<0.05$.

\section{Results}

\section{Effects of ART on chromosomal radiosensitivity in HIV positive individuals}

The results of the MN assay for the four groups (HIV patients not on treatment, HIV individuals on ART regimens TDF+3TC+EFV and $\mathrm{D} 4 \mathrm{~T}+3 \mathrm{TC}+\mathrm{EFV}$ and healthy controls) are shown in Table 2. In unirradiated cells (0Gy), there were no significant differences in spontaneous mean MN yields between the groups. Radiation-induced $\mathrm{MN}$ yields were calculated by subtracting spontaneous $\mathrm{MN}$ yields from MN yields in irradiated cells. The radiation-induced MN yields were significantly higher in patients (both regimens and without ART) compared to healthy controls at both $2 \mathrm{~Gy}$ and $4 \mathrm{~Gy}$. Although both patient groups on ART had higher MN yields than HIV patients not on treatment, patients on $\mathrm{TDF}+3 \mathrm{TC}+\mathrm{EFV}$ had the highest average MN yields, the increase being significant at 4Gy. The HIV patients without ART had significantly lower CD4 counts compared to those on ART. There was no correlation between CD4 counts, time on ART treatment and MN yields (data not shown). The mean NDI values for the unirradiated (0Gy) cultures were similar for all the groups, but the mean NDI for the irradiated cultures (2Gy and 4Gy) were significantly lower in the patients on ART compared to the HIV patients without ART and the healthy controls ( $\mathrm{p}<0.005)$.

\begin{tabular}{|l|l|l|l|l|l|}
\hline & & & & HIV ART & HIV ART \\
\hline & $\begin{array}{l}\text { Healthy } \\
\text { individuals }\end{array}$ & $\begin{array}{l}\text { HIV } \\
\text { without } \\
\text { ART }\end{array}$ & D4T/3TC/EFV & TDF/3TC/EFV \\
\hline 0Gy & N & 20 & 20 & 19 & 20 \\
\hline & Mean & 14 & 14 & 12 & 10 \\
\hline & SD & 6.4 & 6.0 & 7.4 & 5.8 \\
\hline $\begin{array}{l}\text { 2Gy } \\
\text { induced }\end{array}$ & N & 20 & 19 & 18 & 18 \\
\hline & Mean & 300 & 355 & 368 & 386 \\
\hline & SD & 58.3 & 71.4 & 58.9 & 58.2 \\
\hline & p values vs controls & 0.0125 & 0.001 & $<0.0001$ \\
\hline & $\begin{array}{l}p \text { values vs HIV } \\
\text { without ART }\end{array}$ & & 0.5414 & 0.1531 \\
\hline
\end{tabular}




\begin{tabular}{|l|l|l|l|l|l|}
\hline $\begin{array}{l}\text { 4Gy } \\
\text { induced }\end{array}$ & $\mathrm{N}$ & 20 & 18 & 16 & 19 \\
\hline & Mean & 842 & 969 & 1018 & 1076 \\
\hline & $\mathrm{SD}$ & 151.6 & 134.6 & 157.8 & 146.6 \\
\hline & $\mathrm{p}$ values vs controls & 0.0098 & 0.0017 & $<0.0001$ \\
\hline & $\begin{array}{l}\mathrm{p} \text { values vs HIV } \\
\text { without ART }\end{array}$ & & 0.3407 & 0.0277 \\
\hline
\end{tabular}

Table 2: MN yields (per 1000BN) for the HIV positive individuals not taking ART, HIV positive individuals taking 2 different ART regimes and healthy individuals.

\section{Effect of ART on chromosomal radiosensitivity of health care workers}

The MN yields for the 10 health care workers on prophylactic ART before starting ART (T1), at the completion of ART (T2), or 3-4 months after the completion of ART (T3) are shown in Table 3. There were no significant differences between the mean spontaneous and radiation-induced $\mathrm{MN}$ yields at the 3 measured time points.

\begin{tabular}{|l|l|l|l|l|}
\hline & & T1 & T2 & T3 \\
\hline 0 Gy & Mean & 10.3 & 13.4 & 10.9 \\
\hline & SD & 3.5 & 5.2 & 4.4 \\
\hline & $p$ values & & 0.234 & 0.836 \\
\hline $\mathbf{2}$ Gy & Mean & 307.7 & 297.1 & 296.1 \\
\hline & SD & 90.9 & 78.8 & 82.7 \\
\hline & $p$ values & & 0.625 & 0.547 \\
\hline 4Gy & Mean & 826.2 & 842.0 & 834.0 \\
\hline & SD & 146.2 & 206.0 & 192.2 \\
\hline & $p$ values & & 0.943 & 0.844 \\
\hline
\end{tabular}

Table 3: Comparison of MN yields (per 1000BN) of health care workers taking prophylactic post-exposure ART before starting ART (T1), after 3-4 weeks on ART (T2) and 3 months after ART (T3).

\section{Discussion}

The present pilot study investigated if HIV and antiretroviral medication has an influence on chromosomal radiosensitivity. This is one of the first studies looking at the effects of ART on radiosensitivity in HIV infected individuals and is potentially very important for many cancer patients and radiation workers in South Africa exposed to radiation that will be HIV positive and on ART. Since NRTIs interact with host DNA, inhibit mammalian DNA polymerases and affect the cell cycle [21], it is possible that they have an impact on DNA damage repair and affect chromosomal radiosensitivity. Some studies have shown antiretroviral drugs to be radioprotective while others have suggested a radiosensitising effect $[25,26]$. For the first part of this study we investigated the chromosomal radiosensitivity of HIV patients not on ART, HIV patients on two common ART regimens $(\mathrm{d} 4 \mathrm{~T}+3 \mathrm{TC}+\mathrm{EFV}$ and $\mathrm{TDF}+3 \mathrm{TC}+\mathrm{EFV})$ and compared it to healthy uninfected controls.
Micronuclei are considered a biomarker of cytotoxicity. In unirradiated cells no difference in $\mathrm{MN}$ yields between the four groups was detected, suggesting no cytotoxicity of the ART in-vivo. These findings are in agreement with those of Robbins et al. [32] who detected no biologically relevant changes in lymphocyte chromosomal aberrations in $26 \mathrm{HIV}$ positive patients on combinations of ART (AZT, 3TC, d4T) across a number of time points. On the contrary, Shafik et al. (1991) [33] observed a high increase in chromosomal aberrations in AIDS patients treated with AZT and suggested that AZT induces genetic damage in lymphocytes of AIDS patients. None of the studied HIV positive patients were taking AZT, so this could explain the discrepancy between Shafik et al. (1991) [33] and the results presented here. Proliferation rates of lymphocytes from HIV infected individuals are known to be higher than HIV uninfected individuals [34], but this was not reflected in the NDI's calculated in this study. In lymphocytes irradiated with 2 and $4 \mathrm{~Gy}$, a difference in $\mathrm{MN}$ between the groups was noted that points to differences in chromosomal radiosensitivity. All HIV patients (those on ART and without ART) had significantly higher MN than healthy controls. This confirms earlier findings of Baeyens et al. [15]. The patients on the ART regimens had higher MN yields than HIV patients not on ART, with the TDF+3TC+EFV regimen presenting with significantly higher radiation-induced $\mathrm{MN}$ at $4 \mathrm{~Gy}$. The results suggest that substitution of $\mathrm{d} 4 \mathrm{~T}$ with TDF in the ART regimen of HIV patients leads to an enhanced chromosomal radiosensitivity. Cytotoxicity studies on TDF in vitro have been conflicting. Cihlar et al. (2002) [35] and Hecht et al. (2013) [36] have shown TDF to exhibit low cytotoxicity in various cell types, while a study by Bruning et al. [23] indicated that TDF induced DNA damage, reduced cell viability and induced cell cycle disturbances leading to apoptosis in cancer cells. This last statement could mean that TDF may be beneficial in radiosensitising tumour cells in HIV positive individuals, but its effect on normal tissue radiotoxicity, seen in the lymphocytes in this study, may be adverse. The significantly higher MN yields at $4 \mathrm{~Gy}$ in the TDF group could reflect the un/misrepaired, severe DNA damage caused by the combination of HIV-infection, TDF and ionising radiation. Since studies have shown an association between clinical response to radiotherapy and chromosomal radiosensitivity [16,17], the effect of $\mathrm{ART}$, especially TDF, on radiotoxicity requires further investigation.

For the second part of the study, we investigated the chromosomal radiosensitivity of 10 non-HIV-infected South African HCWs on the post-exposure prophylaxis ART to determine the effect of ART on chromosomal radiosensitivity without HIV as a confounding factor. All participants were on an ART regimen including two NRTIs. In unirradiated lymphocytes of these donors, no differences were detected in average MN yields before, during or after ART. The increase in chromosomal aberrations in AIDS patients taking AZT, seen in the study of Shafik et al. [33], was not noted in the HCWs taking AZT. The different results between both studies could suggest that not only AZT but the combination of HIV infection and AZT lead to increased chromosomal damage. The in vitro genotoxicity effects of AZT and 3TC seen in studies of Stern et al. [29], Lourenco et al. [28], and Gonzalez Cid et al. [30] were not confirmed in the current study. The fact that in these studies, ART was added in high concentrations for short terms in vitro to cell cultures, while in this study the ART was administered in vivo over a longer period, could explain the observed difference. No changes were noted in average radiation-induced MN yields of the HCWs at the different time points. Jagetia and Aruna [25], found that administration of AZT to Hela cells before exposure to different doses of $\gamma$-radiation resulted in a significant elevation in 
the yield of MN. This was in contrast to Copaceanu et al. [26] who suggested radioprotection of AZT in Hela cell lines exposed to irradiation in-vitro. Several publications demonstrated that AZT and 3TC alter cell cycle kinetics and increase the proportion of cells in S phase [21,22]. Radiosensitivity of cells differs according to cell type and phase of the cell cycle the cells are in at the time of irradiation [37]. In both studies of Jagetia and Aruna [25] and Copaceanu et al. [26] irradiations were performed on rapidly-dividing Hela cell lines, while in the current study, lymphocytes were irradiated in the G0 phase of the cell cycle.

Following from the MN data on HCWs taking ART, we could not support that either AZT or 3TC have a radiosensitising or radioprotective effect on lymphocytes of healthy individuals. The fact that the increased chromosomal radiosensitivity, seen in HIV positive individuals on ART was not observed in the lymphocytes of HCWs on ART could be due to the following reasons: 1) The duration of the ART was different (more than 6 months for HIV positive patients versus 3-4 weeks in HCWs). 2) It is the ART and the HIV virus in combination with the radiation that led to increased $\mathrm{MN}$ yields in HIV-infected patients on ART. 3) It is different ART compounds or the combinations (d4T, TDF and EFV versus AZT) exerting different effects in the cells in response to ionizing radiation.

The results of this study suggest that HIV and certain recent ART $(\mathrm{TDF}+3 \mathrm{TC}+\mathrm{EFV})$ given to HIV positive individuals can lead to enhanced lymphocyte chromosomal radiosensitivity and therefore may impose higher risks for radiation-induced normal tissue complications in these patients. Further investigation of the different compounds of ART in combination with ionizing radiation is warranted, as HIV positive cancer patients undergoing radiotherapy and HIV positive radiation workers may potentially benefit from having their ART regimens altered according to the radiomodulatory effects of the various drugs.

\section{Acknowledgement}

This research was supported by 'iThemba LABS' South Africa, Nuclear Technologies in Medicine and the Biosciences Initiative (NTeMBI), a national technology platform developed and managed by the South African Nuclear Energy Corporation (NECSA) and a 'VLIR Own Initiative Programme' between Belgium and South Africa (ZEIN2011PR387). The authors wish to thank all donors who participated in this study. We also thank the nurses at Themba Lethu HIV unit at the Helen Joseph Hospital in Johannesburg for the assistance in sample collection.

\section{References}

1. STATSSA (2013) Mid-year population estimates South Africa.

2. UNAIDS (2014) The GAP report 2014. In. Geneva.

3. WHO (2006) Antiretroviral therapy for HIV infection in adults and adolescents.

4. Renaud-Théry F, Avila-Figueroa C, Stover J, Thierry S, Vitoria M, et al. (2011) Utilization patterns and projected demand of antiretroviral drugs in low- and middle-income countries. AIDS Res Treat 2011: 749041.

5. WHO (2010) Antiretroviral therapy for HIV infection in adults and adolescents.

6. SADOH (2010) Clinical Guidelines for the Management of HIV \& AIDS in Adults and Adolescents.

7. WHO (2013) Consolidated guidelines on the use of antiretroviral drugs for treating and preventing HIV infection.
8. Davies N (2013) Fixed-dose combination for adults accessing antiretroviral therapy. South African Journal of HIV Medicine 14: 41-43.

9. Cardo DM, Culver DH, Ciesielski CA, Srivastava PU, Marcus R, et al. (1997) A case-control study of HIV seroconversion in health care workers after percutaneous exposure. Centers for Disease Control and Prevention Needlestick Surveillance Group. N Engl J Med 337: 1485-1490.

10. Karstaedt AS, Pantanowitz L (2001) Occupational exposure of interns to blood in an area of high HIV seroprevalence. S Afr Med J 91: 57-61.

11. WHO (2007) Joint WHO/ILO guidelines on post-exposure prophylaxis (PEP) to prevent HIV infection.

12. CDC (1993) Revised Classification System for HIV Infection and Expanded Surveillance Case Definition for AIDS Among Adolescents and Adults. In: Centers for Disease control.

13. Bonnet F, Burty C, Lewden C, Costagliola D, May T, et al. (2009) Changes in cancer mortality among HIV-infected patients: the Mortalité 2005 Survey. Clin Infect Dis 48: 633-639.

14. Crum-Cianflone N, Hullsiek KH, Marconi V, Weintrob A, Ganesan A, et al. (2009) Trends in the incidence of cancers among HIV-infected persons and the impact of antiretroviral therapy: a 20-year cohort study. AIDS 23: 41-50.

15. Baeyens A, Slabbert JP, Willem P, Jozela S, Van Der Merwe D, et al. (2010) Chromosomal radiosensitivity of HIV positive individuals. Int J Radiat Biol 86: 584-592.

16. Lee TK, Allison RR, O'Brien KF, Johnke RM, Christie KI, et al. (2003) Lymphocyte radiosensitivity correlated with pelvic radiotherapy morbidity. Int J Radiat Oncol Biol Phys 57: 222-229.

17. Sprung CN, Chao M, Leong T, McKay MJ (2005) Chromosomal radiosensitivity in two cell lineages derived from clinically radiosensitive cancer patients. Clin Cancer Res 11: 6352-6358.

18. Housri N, Yarchoan R, Kaushal A (2010) Radiotherapy for patients with the human immunodeficiency virus: are special precautions necessary? Cancer 116: 273-283.

19. Sankatsing SU, Hillebregt MM, Gras L, Brinkman K, van der Ende M, et al. (2013) Prolonged decrease of CD4+ T lymphocytes in HIV-1-infected patients after radiotherapy for a solid tumor. J Acquir Immune Defic Syndr 62: 546-549.

20. Munch-Petersen B (1988) Azidothymidine inhibits mitogen stimulated growth and DNA-repair in human peripheral lymphocytes. Biochem Biophys Res Commun 157: 1369-1375.

21. Fang JL, McGarrity LJ, Beland FA (2009) Interference of cell cycle progression by zidovudine and lamivudine in NIH $3 \mathrm{~T} 3$ cells. Mutagenesis 24: 133-141.

22. Olivero OA, Tejera AM, Fernandez JJ, Taylor BJ, Das S, et al. (2005) Zidovudine induces S-phase arrest and cell cycle gene expression changes in human cells. Mutagenesis 20: 139-146.

23. Brüning A, Burger P, Gingelmaier A, Mylonas I (2012) The HIV reverse transcriptase inhibitor tenofovir induces cell cycle arrest in human cancer cells. Invest New Drugs 30: 1389-1395.

24. Gomez DE, Tejera AM, Olivero OA (1998) Irreversible telomere shortening by 3'-azido-2',3'-dideoxythymidine (AZT) treatment. Biochem Biophys Res Commun 246: 107-110.

25. Jagetia GC, Aruna R (2003) Correlation of micronuclei-induction with the cell survival in HeLa cells treated with a base analogue, azidothymidine (AZT) before exposure to different doses of gammaradiation. Toxicol Lett 139: 33-43.

26. Copaceanu ML, Coucke PA, Cottin E, Paschoud N, Mirimanoff RO (1995) Azidothymidine (AZT) as a potential modifier of radiation response in vitro. Acta Oncol 34: 213-218.

27. Norppa H, Falck GC (2003) What do human micronuclei contain? Mutagenesis 18: 221-233.

28. Lourenco ED, do Amaral VS, Lehmann M, Dihl RR, Schmitt VM, et al. (2010) Micronuclei induced by reverse transcriptase inhibitors in mononucleated and binucleated cells as assessed by the cytokinesis-block micronucleus assay. Genet Mol Biol 33:756-760. 
Citation: Herd O, Francies F, Slabbert J, Baeyens A (2014) The Effect of HIV and Antiretroviral Therapy on Chromosomal Radiosensitivity. J

Page 6 of 6

29. Stern M, Cid MG, Larripa I, Slavutsky I (1994) AZT-induction of micronuclei in human lymphocyte subpopulations. Toxicol Lett 70 : 235-242.

30. González Cid M, Larripa I (1994) Genotoxic activity of azidothymidine (AZT) in in vitro systems. Mutat Res 321: 113-118.

31. Fenech M (1993) The cytokinesis-block micronucleus technique: a detailed description of the method and its application to genotoxicity studies in human populations. Mutat Res 285: 35-44.

32. Robbins WA, Witt KL, Haseman JK, Dunson DB, Troiani L, et al. (2001) Antiretroviral therapy effects on genetic and morphologic end points in lymphocytes and sperm of men with human immunodeficiency virus infection. J Infect Dis 184: 127-135.

33. Shafik HM, Nokta MA, Pollard RB (1991) Recombinant human interferon beta ser protects against zidovudine-induced genetic damage in AIDS patients. Antiviral Res 16: 205-212.
34. Trautmann L, Mbitikon-Kobo FM, Goulet JP, Peretz Y, Shi Y, et al. (2012) Profound metabolic, functional, and cytolytic differences characterize HIV-specific CD8 T cells in primary and chronic HIV infection. Blood 120: 3466-3477.

35. Cihlar T, Birkus G, Greenwalt DE, Hitchcock MJ (2002) Tenofovir exhibits low cytotoxicity in various human cell types: comparison with other nucleoside reverse transcriptase inhibitors. Antiviral Res 54: 37-45.

36. Hecht M, Harrer T, Büttner M, Schwegler M, Erber S, et al. (2013) Cytotoxic effect of efavirenz is selective against cancer cells and associated with the cannabinoid system. AIDS 27: 2031-2040.

37. Jeggo P, Lavin MF (2009) Cellular radiosensitivity: how much better do we understand it? Int J Radiat Biol 85: 1061-1081. 